\title{
Exogenous and Endogenous Triggers Differentially Stimulate Pigr Expression and Antibacterial Secretory Immunity in the Murine Respiratory Tract
}

\author{
Alexander Pausder ${ }^{1,2,3} \cdot$ Jennifer Fricke $e^{4,9} \cdot$ Klaus Schughart $^{4,5,6}$ (D) Jens Schreiber ${ }^{7}$ - Till Strowig $^{8}$ (D) \\ Dunja Bruder ${ }^{1,2}$ (1) . Julia D. Boehme ${ }^{1,2}$ (1)
}

Received: 27 July 2021 / Accepted: 15 November 2021 / Published online: 26 November 2021

(C) The Author(s) 2021

\begin{abstract}
Purpose Transport of secretory immunoglobulin A (SIgA) through the airway epithelial cell barrier into the mucosal lumen by the polymeric immunoglobulin receptor (pIgR) is an important mechanism of respiratory mucosal host defense. Identification of immunomodulating substances that regulate secretory immunity might have therapeutic implications with regard to an improved immune exclusion.

Thus, we sought to analyze secretory immunity under homeostatic and immunomodulating conditions in different compartments of the murine upper and lower respiratory tract (URT\&LRT).

Methods Pigr gene expression in lung, trachea, and nasal-associated lymphoid tissue (NALT) of germ-free mice, specific pathogen-free mice, mice with an undefined microbiome, as well as LPS- and IFN- $\gamma$-treated mice was determined by quantitative real-time PCR. IgA levels in bronchoalveolar lavage (BAL), nasal lavage (NAL), and serum were determined by ELISA. LPS- and IFN- $\gamma$-treated mice were colonized with Streptococcus pneumoniae and bacterial CFUs were determined in URT and LRT.

Results Respiratory Pigr expression and IgA levels were dependent on the degree of exposure to environmental microbial stimuli. While immunostimulation with LPS and IFN- $\gamma$ differentially impacts respiratory Pigr expression and IgA in URT $v s$. LRT, only prophylactic IFN- $\gamma$ treatment reduces nasal colonization with $S$. pneumoniae.

Conclusion Airway-associated secretory immunity can be partly modulated by exposure to microbial ligands and proinflammatory stimuli. Prophylactic IFN- $\gamma$-treatment modestly improves antibacterial immunity in the URT, but this does not appear to be mediated by SIgA or $\mathrm{pIgR}$.
\end{abstract}

Keywords Secretory immunity $\cdot$ Respiratory tract $\cdot$ Polymeric immunoglobulin receptor $\cdot$ Immune modulation $\cdot$ Infection

Julia D. Boehme

julia.boehme@med.ovgu.de

1 Research Group Infection Immunology, Institute of Medical Microbiology and Hospital Hygiene, Health Campus Immunology, Infectiology and Inflammation, Otto-Von-Guericke-University, Leipziger Strasse 44, 39120 Magdeburg, Germany

2 Research Group Immune Regulation, Helmholtz Centre for Infection Research, Braunschweig, Germany

3 ESF Graduate School ABINEP, Magdeburg, Germany

4 Research Group Infection Genetics, Helmholtz Centre for Infection Research, Braunschweig, Germany
5 University of Veterinary Medicine, Hannover, Germany

6 University of Tennessee Health Science Center, Memphis, TN, USA

7 Experimental Pneumology, Health Campus Immunology, Infectiology and Inflammation, University Hospital for Pneumology, Otto-Von-Guericke-University, Magdeburg, Germany

8 Department of Microbial Immune Regulation, Helmholtz Centre for Infection Research, Braunschweig, Germany

9 Current Address: Research Group Nanoinfection Biology, Helmholtz Centre for Infection Research, Braunschweig, Germany 


\section{Introduction}

Airway epithelial cells (AECs) constitute the first line of defense against respiratory pathogens. They express transmembrane proteins, which form tight junctions that allow only small ions or water to traverse paracellularly [1]. Claudin and occludin are vital for epithelial defense [2] and altered claudin expression affects airway epithelial barrier function [3, 4]. Furthermore, AECs constitutively secrete antimicrobial proteins, complement factors and cytokines, and rapidly mount antimicrobial immune responses upon inflammatory and infectious stimuli [5-9]. Importantly, AECs have a central function in antibodymediated mucosal immunity. Multimeric $\operatorname{IgA}$ and $\operatorname{IgM}$ are actively transported through AECs via the polymeric immunoglobulin receptor (PIGR) and are secreted into the mucosal lumen as secretory immunoglobulins (SIgs) [10]. Especially $\mathrm{SIgA}$ is known to prevent pathogen adhesion, thus averting microbial infiltration [11]. Moreover, SIgA plays a crucial role in the regulation of Streptococcus pneumoniae nasal colonization in mice [12].

In this context, Pigr deficiency manifests in susceptibility to mycobacterial respiratory infections [13] and development of a COPD-like phenotype driven by an altered lung microbiome and bacterial invasion of the airway epithelium [14]. The importance of SIgA for airway homeostasis is furthermore highlighted by the findings of SIgA deficiency in small airways of COPD patients, which is associated with persistent inflammation and airway wall remodeling [15]. Moreover, chronic airway diseases reduce PIGR expression in the bronchial epithelium resulting in increased disease severity (COPD) and impaired SIgA-mediated mucosal defense (asthma) [16, 17]. While the key role of PIGR and secretory immunity for airway homeostasis is undisputable, knowledge on their expression and regulation in the airways is still fragmentary. Since targeted modulation of secretory immunity represents an interesting option to improve immune exclusion of respiratory pathogens, we here aimed to further dissect PIGR-mediated immunity in the airways with the specific focus on the applicability of exogenous and endogenous stimuli to regulate this aspect of humoral antimicrobial defense.

\section{Methods}

\section{Mice}

BALB/c and C57BL/6 J mice (age: 11-46 weeks) were maintained in individually ventilated cages (IVCs) under specific pathogen-free (SPF) conditions at the Helmholtz Centre for Infection Research (HZI), Braunschweig. Germfree mice (C57BL/6 N, age: 10 weeks) were bred and maintained in isolators in a germ-free (GF) facility (HZI). C57BL/6 J mice with an undefined microbiome (maintained in open cages, age: $10-18$ weeks) were provided by Dirk Schlüter (Otto-von-Guericke-University [OvGU], Magdeburg). For pneumococcal colonization experiments female C57BL/6JRj mice (age: 12 weeks) were purchased from Janvier Labs (France) and maintained in IVCs under SPF conditions (OvGU).

\section{Treatment with Immunomodulating Substances}

BALB/c, C57BL/6 J, and C57BL/6JRj mice were treated intranasally (i.n.) with LPS (Sigma-Aldrich, Germany), $(10 \mu \mathrm{g} / 25 \mu \mathrm{l}$ PBS or solvent alone) or recombinant murine

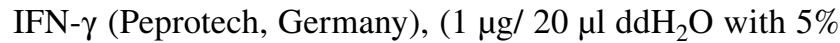
$\mathrm{BSA}$ or solvent alone). BALB/c and C57BL/6 J mice were sacrificed 1 or 2 days post-treatment. Lung, trachea, NALT, BAL, NAL, and serum were collected. Organs were used for RNA isolation and qPCR. Fluids were used for ELISA analysis. Blood was collected by cardiac puncture. BAL fluid was collected by flushing the lungs with $1 \mathrm{ml}$ PBS via the trachea. The nasopharynx was flushed with $1 \mathrm{ml}$ PBS via the trachea and NAL fluid was collected at the nostrils.

\section{Pneumococcal Infection}

Streptococcus pneumoniae serotype 19F (strain BHN100) [18] was provided by Birgitta Henriques-Normark (Karolinska Institutet, Stockholm). Bacteria were grown in ToddHewitt yeast (THY) medium as previously described [19]. LPS- and IFN- $\gamma$-treated C57BL/6JRj mice and control groups were infected i.n. with $10^{8} \mathrm{~S}$. pneumoniae $19 \mathrm{~F}$ in $10 \mu \mathrm{l}$ PBS $48 \mathrm{~h}$ after the first treatment. Mice were sacrificed $18 \mathrm{~h}$ post-infection and lung, trachea, NALT, and nasopharynx were homogenized using a tissue homogenizer (KINEMATICA AG, Switzerland). Samples were plated onto Columbia blood agar plates (BD Diagnostic Systems, Germany) and incubated over night at $37^{\circ} \mathrm{C}, 5 \% \mathrm{CO}_{2}$. CFU were counted to determine the bacterial burden.

\section{Quantitative Real-Time PCR (qPCR)}

RNA was isolated from lung, trachea, NALT, and MLE15 cells using RNeasy Plus Mini Kit (QIAGEN, Germany). cDNA was synthesized from $1 \mu \mathrm{g}$ of RNA using Oligo dT Primers (Thermo Fisher Scientific, USA), Random Primers (Thermo Fisher Scientific, USA), dNTP-Mix (10 mM), and SuperScript ${ }^{\mathrm{TM}}$ III Reverse Transcriptase (Thermo Fisher Scientific, USA). QPCR was performed using the SensiFAST ${ }^{\text {TM }}$ SYBR® No-ROX Kit (Bioline, USA). Temperature profile: 
$95{ }^{\circ} \mathrm{C}$ for $2 \mathrm{~min}, 40$ cycles at $95^{\circ} \mathrm{C}$ for $5 \mathrm{~s}, 60{ }^{\circ} \mathrm{C}$ for $10 \mathrm{~s}$, and $72{ }^{\circ} \mathrm{C}$ for $5 \mathrm{~s}$. B-Actin (Actb) served as reference gene. Primer sequences: Pigr forward: 5'-GTGCCCGAAACT GGATCACC-3', Pigr reverse: 5'-TGGAGACCCCTGAAA AGACAGT-3', Actb forward: 5'-ACACCCGCCACCAGT TCG-3', Actb reverse: 5'-GTCACCCACATAGGAGTC CTTC-3', Cldn-7 forward: 5'-AGCGAAGAAGGCCCG AATAG-3', $C l d n-7$ reverse: 5'-AGGTCCAAACTCGTACTT AACG-3', $C l d n$ - 18 forward: 5'-GACACCAGATGACAG CAACTTC-3', Cldn-18 reverse: 5'-TTCATCGTCTTCTGT GCGGG-3', IgJ forward: 5'-GCATGTGTACCCGAGTTA CC-3', IgJ reverse: 5'-TTCAAAGGGACAACAATTCGG-3', and $C D 19$ forward: 5'-CCTGGGCATCTTGCTAGTGA-3', CD19 reverse: 5'-CGGAACATCTCCCCACTATCC-3'. Expression of target genes in relation to reference gene was determined using the $2^{-\Delta \Delta C T}$ method.

\section{Enzyme-Linked Immunosorbent Assay (ELISA)}

Relative IgA levels (including monomeric IgA, dimeric IgA, and SIgA molecules) in BAL, NAL, and serum were determined by ELISA using a monoclonal rat anti-mouse IgA capture antibody (Southern Biotech, USA) in combination with a polyclonal rabbit anti-mouse IgA secondary antibody (Abcam, UK) and a polyclonal swine anti-rabbit, HRPOlinked detection antibody (Dako, UK).

\section{In Vitro Stimulation}

MLE-15 cells were cultivated in Dulbecco's Modified Eagle's Medium (DMEM, Gibco, USA) supplemented with $4.5 \mathrm{~g} / \mathrm{l}$ glucose, $10 \%$ FBS (Biowest, USA), and $1 \%$ penicillin/streptomycin (Gibco, USA). $3 \times 10^{5}$ cells were seeded in 12-well plates and incubated in $1 \mathrm{ml}$ medium over night at $37{ }^{\circ} \mathrm{C}, 5 \% \mathrm{CO}_{2}$. Cells were washed using $1 \mathrm{ml}$ DMEM (4.5 g/l glucose, no additives). Recombinant murine IFN- $\gamma$ (Peprotech, Germany) was diluted in DMEM (w/o additives) and added to the cells. After $24 \mathrm{~h}$ supernatants were removed and RNA was isolated from the cells and used for qPCR.

\section{Statistical Analysis}

Statistical analyses were performed either by two-tailed, unpaired t test (Gaussian distribution, two groups), Mann-Whitney test (no Gaussian distribution, two groups), one-way ANOVA (Gaussian distribution, more than two groups, post-test: Bonferroni's Multiple Comparison Test), or Kruskal-Wallis test (no Gaussian distribution, more than two groups, post-test: Dunn's Multiple Comparison Test) using GraphPad Prism software (GraphPad Software Inc., USA, Version 5.04).

\section{Results}

In order to determine differences in Pigr gene expression in the upper (URT) and lower respiratory tract (LRT) and its dependency on genetic background, sex, and age, we initially compared Pigr expression patterns in commonly used mouse strains and different sexes. BALB/c (Fig. 1a) as well as C57BL/6 J mice (Fig. 1b) exhibit highest Pigr expression in the trachea, followed by nasal-associated lymphoid tissue (NALT) and lung. While pulmonary Pigr expression levels were relatively constant, higher variations were detected in trachea and NALT. Moreover, comparative analyses of Pigr expression in BALB/c vs. C57BL/6 J mice (Fig. S1a) and male vs. female C57BL/6 J mice (Fig. S1b) revealed no significant differences. We also analyzed whether Pigr gene a

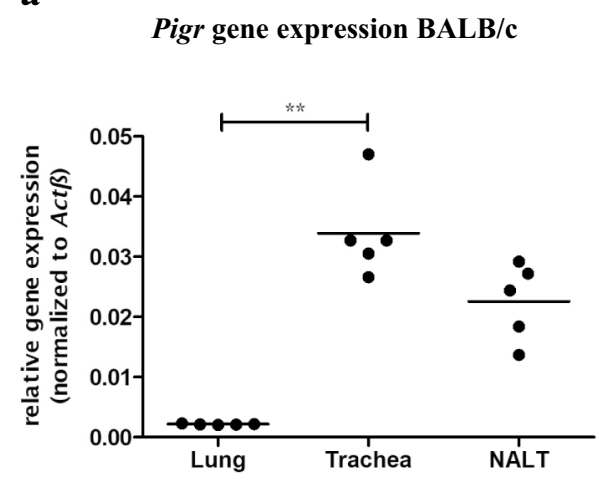

Fig. 1 Basal Pigr expression in the airways. RNA from lung tissue, trachea, and nasal-associated lymphoid tissue (NALT) of a $\mathrm{BALB} / \mathrm{c}$ mice $(n=5)$ and $\mathbf{b} \mathrm{C} 57 \mathrm{BL} / 6 \mathrm{~J}$ mice $(n=14)$ was isolated and reversely transcribed into cDNA. Pigr expression was assessed b

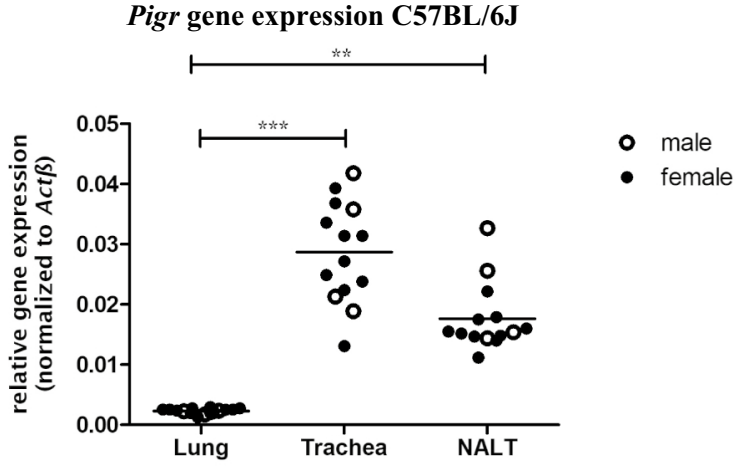

by qPCR. Actb served as reference gene (data for individual mice are graphed; mean is indicated by horizontal line; ** for $p \leq 0.01$; *** for $p \leq 0.001)$ 
expression is age-dependent (Fig. S2a-c) but found no such correlations. Together, these data demonstrate marked tissue-specific differences in Pigr gene expression, which were however independent of genetic background, sex, and age.

To determine whether Pigr expression and secretory immunity in the airways were influenced by microbial exposure we compared germ-free (GF) mice (no microbial exposure), SPF mice (IVCs, exposure to a limited microbial flora), and mice with an undefined microbiome (open cage maintenance, highest degree of exposure to airborne microorganisms). While similar Pigr expression levels were observed in the LRT of all three experimental groups, we detected significantly lower Pigr expression in the NALT of mice with an undefined microbiome compared to SPF mice (Fig. 2a). In contrast to the unaltered (lung, trachea) or even reduced (NALT) Pigr expression levels in mice with an undefined microbiome, we detected significantly increased IgA concentration in the LRT (Fig. 2b) and URT (Fig. 2c) in this group, which was associated with a systemic $\operatorname{IgA}$ increase (Fig. 2d).

Since in the intestine IgA production and Pigr expression are induced by microbial components [20-22], we investigated whether intranasal (i.n.) treatment of mice with LPS would affect airway Pigr expression and IgA levels. While Pigr expression in nose and trachea was not affected, we detected significantly increased expression in lung tissue
$48 \mathrm{~h}$ after LPS treatment (Fig. 3a). Interestingly and in discordance with the increased pulmonary Pigr expression IgA levels in BAL and NAL fluid significantly decreased after LPS treatment. The amount of $\operatorname{Ig} \mathrm{A}$ in serum was however not affected (Fig. 3b). We thus speculated that LPS altered the barrier function of AECs, resulting in a decreased epithelial leakage. Therefore, we analyzed $\mathrm{Cldn}$ gene expression in lung and NALT. However, no significant differences in lung or NALT became apparent upon LPS treatment (Fig. 3c, d).

Next to LPS, Interferon- $\gamma$ (IFN- $\gamma$ ) was shown to regulate human PIGR gene expression [23] and we confirmed the Pigr-inducing potential of this cytokine in murine AECs (Fig. S3). To assess possible effects of IFN- $\gamma$ on PIGR-mediated secretory immunity in vivo, we analyzed airway secretory immunity in IFN- $\gamma$-treated mice. While IFN- $\gamma$ did not affect Pigr expression after 48 h (Fig. 4a), the amount of pulmonary IgA increased after IFN- $\gamma$ treatment. IgA levels in NAL and serum were however unaffected (Fig. 4b). We tested whether epithelial leakage might underlie the increased IgA levels by measuring Cldn gene expression in lung and NALT. Cldn gene expression was however not affected by single IFN- $\gamma$ treatment (Fig. 4c, d). To investigate whether increased pulmonary Ig A might arise from Ig-producing B cells which were induced by IFN- $\gamma$, we determined gene expression of the joining chain $(I g J)$ of multimeric $\operatorname{IgA}$ and $\operatorname{IgM}$ in lung

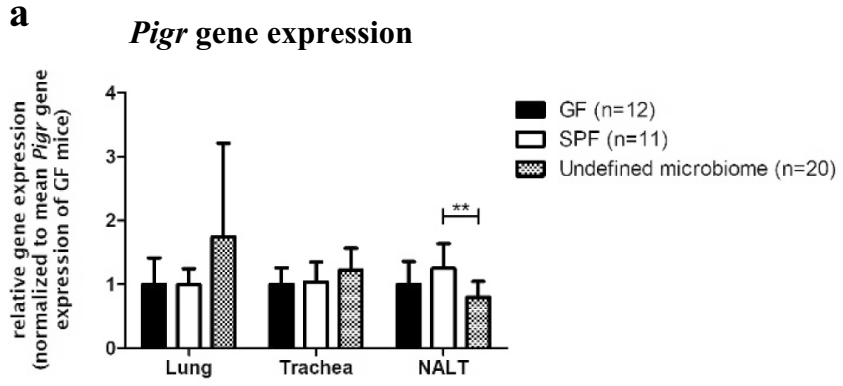

b

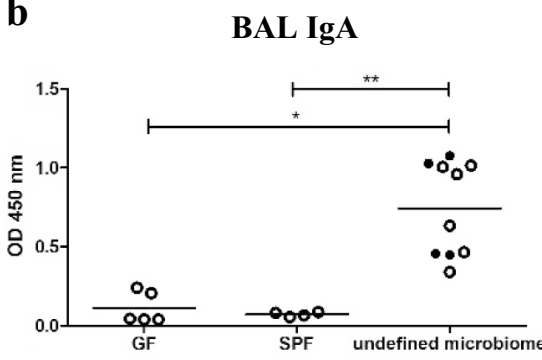

c

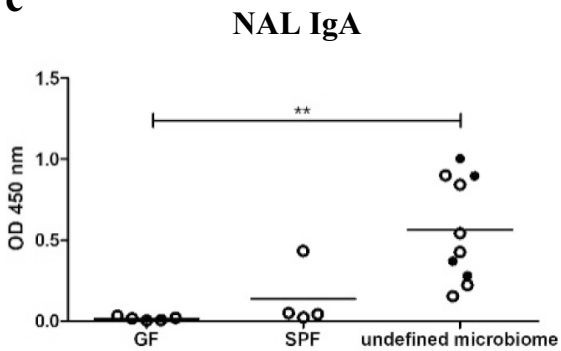

d

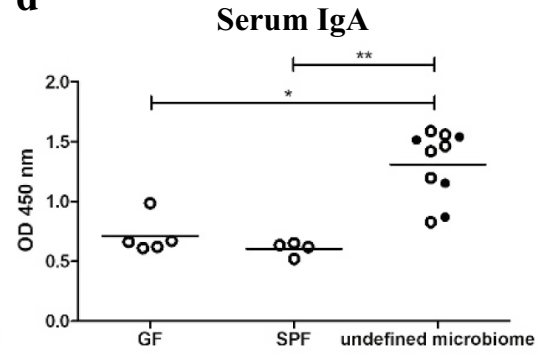

Fig. 2 Pigr expression and secretory immunity in differentially colonized mice. RNA from lung tissue, trachea, and NALT of specific pathogen-free (SPF), germ-free (GF), and mice with an undefined microbiome was isolated and reversely transcribed into cDNA and qPCR analysis was performed $(-$ indicates female, $\bigcirc$ indicates male). a Pigr expression normalized to Actb. Normalized gene expression values of each organ were divided by the mean gene expression of the GF mice for the respective organ (mean expression values \pm SD are graphed). IgA levels were determined in $\mathbf{b}$ bronchoalveolar lavage (BAL), $\mathbf{c}$ nasal lavage (NAL), and $\mathbf{d}$ serum of GF $(n=5)$, SPF $(n=4)$, and mice with an undefined microbiome $(n=10)$ by semi-quantitative ELISA (data for individual mice are graphed; mean is indicated by horizontal line; * for $p \leq 0.05$; ** for $p \leq 0.01$ ) 
a

Pigr gene expression

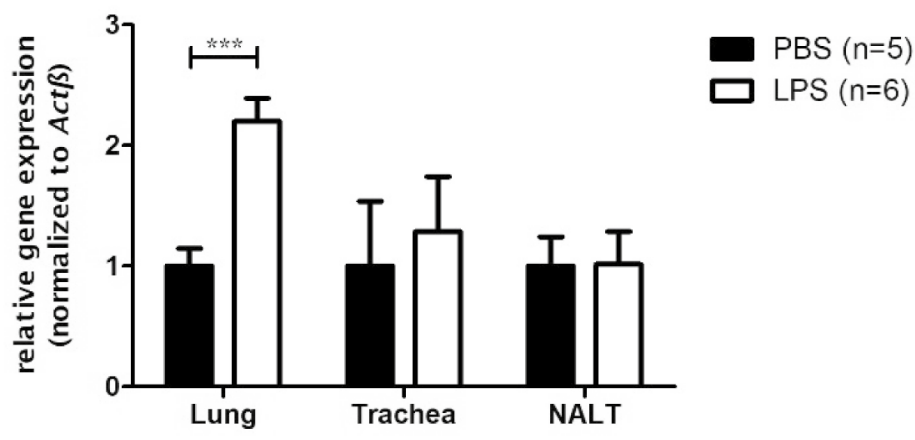

b

BAL IgA

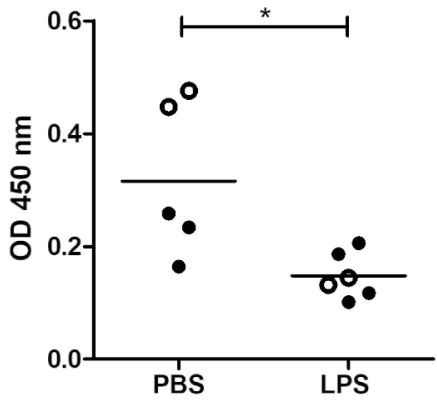

c

Lung Cldn18 gene expression

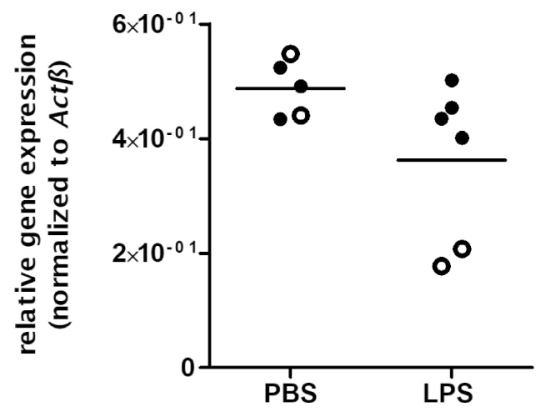

Fig. 3 Effect of LPS treatment on airway secretory immunity and epithelial barrier function. BALB/c mice were treated i.n. with $10 \mu \mathrm{g}$ of LPS $(n=6)$ or solvent alone $(n=5) .48 \mathrm{~h}$ post-treatment RNA from lung tissue, trachea, and NALT was isolated and reversely transcribed into cDNA and qPCR analysis was performed indicates female, $\bigcirc$ indicates male). a Pigr expression normalized to Actb. Normalized Pigr expression values of each organ were divided by the mean Pigr

and NALT. Nevertheless, airway $I g J$ expression was unaltered upon cytokine treatment (Fig. 4e, f), indicating that increased airway IgA levels are most likely not due to an IFN- $\gamma$-mediated increase of Ig-producing cells.
NAL IgA

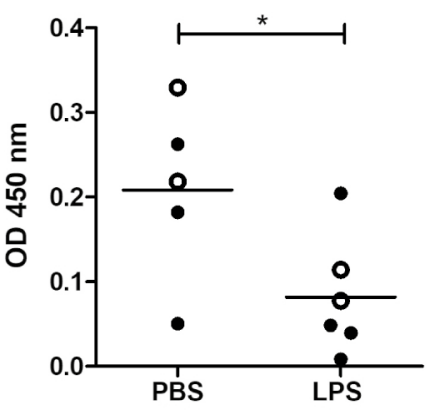

d
Serum IgA

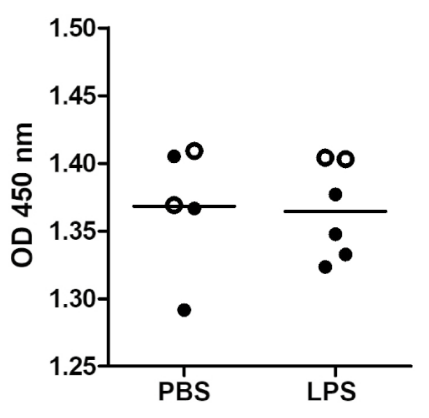

NALT Cldn 7 gene expression

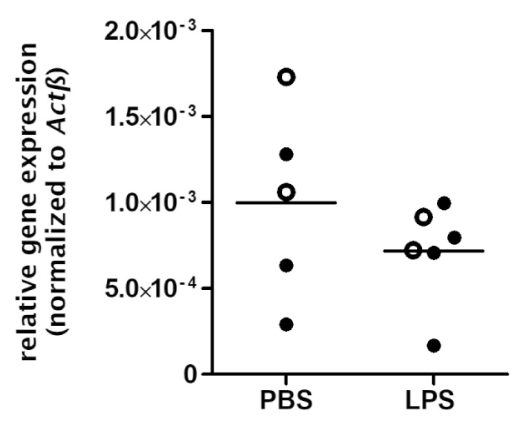

expression of the PBS-treated mice for the respective organ (mean expression values \pm SD are graphed). b IgA levels were determined in BAL, NAL, and serum by semi-quantitative ELISA. c Cldn18 expression normalized to Actb and $\mathbf{d}$ Cldn7 expression normalized to Actb (cumulative data from two experiments; data for individual mice are graphed; mean is indicated by horizontal line; * for $p \leq 0.05$; *** for $p \leq 0.001)$

We finally tested whether modulation of airway secretory immunity by LPS- and IFN- $\gamma$ treatment would ultimately affect antimicrobial defense. To this end, mice were i.n. treated with a single dose of LPS or IFN- $\gamma$. Two days 


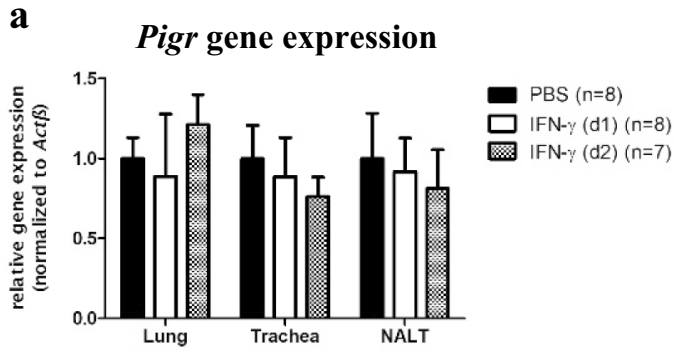

b
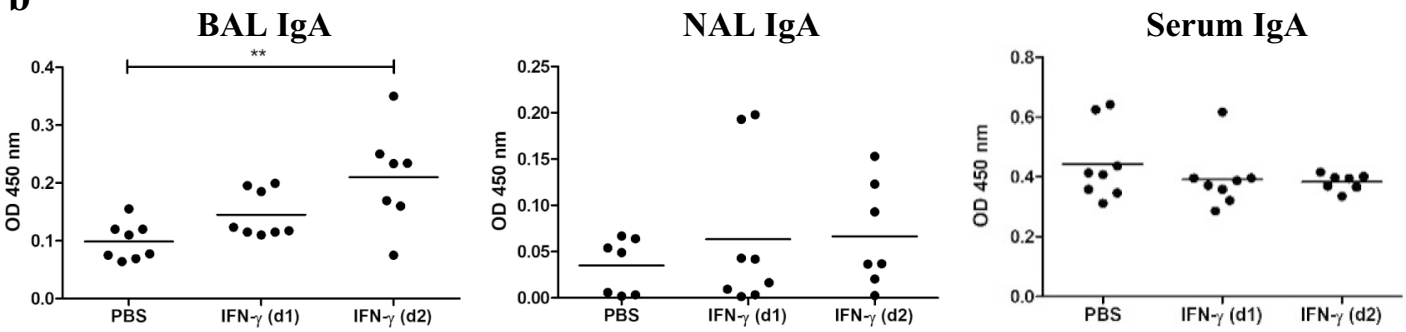

c Lung Cldn18 gene expression

d NALT Cldn7 gene expression
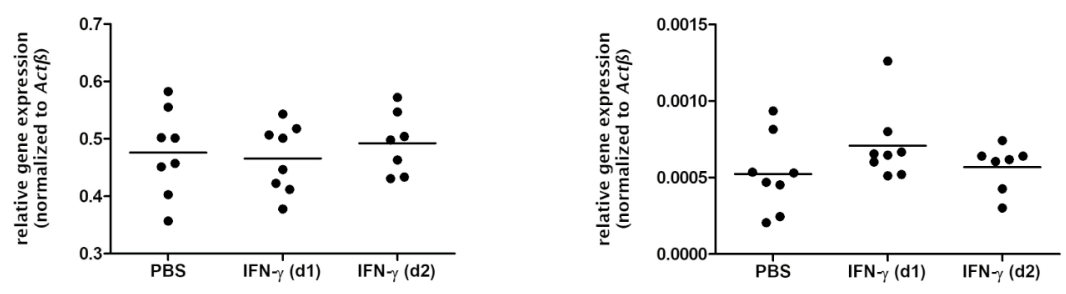

e

Lung Ig $J$ gene expression

\section{f}

NALT IgJ gene expression
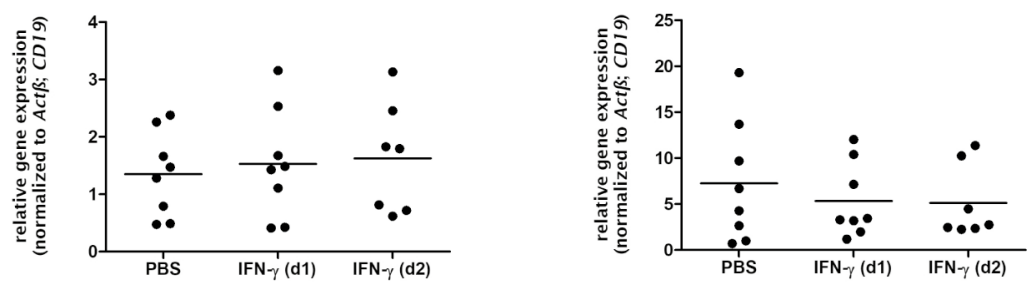

Fig. 4 Effect of IFN- $\gamma$ treatment on secretory immunity, epithelial barrier function, and B cells. BALB/c and $\mathrm{C} 57 \mathrm{BL} / 6 \mathrm{~J}$ mice were treated i.n. with $1 \mu \mathrm{g}$ of IFN- $\gamma(n=8$ for $\mathrm{d} 1, n=7$ for $\mathrm{d} 2)$ or solvent alone $(n=8)$. Lung, trachea, and NALT were removed on $\mathrm{d} 1$ or $\mathrm{d} 2$ post-treatment, RNA was isolated and reversely transcribed into cDNA, and qPCR analysis was performed. a Pigr expression normalized to Actb. Normalized Pigr expression values of each organ were divided by the mean Pigr expression of the PBS-treated group for the

post-treatment mice were inoculated with a colonizing strain of $S$. pneumoniae serotype $19 \mathrm{~F}$ and airway bacterial burden was assessed (Fig. 5a). As expected, no pneumococci were detected in the lung tissue. While LPS treatment did not affect pneumococcal colonization (Fig. 5b), IFN- $\gamma$ treatment led to significantly decreased nasal bacterial burden (Fig. 5c). These results demonstrate that at least in the URT mucosal immunity can be augmented by prophylactic IFN- $\gamma$ treatment. respective organ (mean expression values $\pm \mathrm{SD}$ are graphed). b IgA levels were determined in BAL, NAL, and serum by semi-quantitative ELISA. c $C l d n 18$ expression normalized to $A c t b, \mathbf{d} C l d n 7$ expression normalized to $A c t b$, and $\mathbf{e}+\mathbf{f} I g J$ gene expression normalized to $A c t b$ and $C D 19$ (cumulative data from two experiments; data for individual mice are graphed; mean is indicated by horizontal line; ** for $p \leq 0.01)$

\section{Discussion}

Previous studies on airway Pigr gene and PIGR protein expression mainly employed in vitro approaches [24, 25] or utilized tissue from patients with chronic respiratory diseases [16, 26-28]. Murine studies were either analyzing respiratory Pigr expression in the context of interleukin treatment $[29,30]$ or exposure to pathogen-associated molecules (Cholera toxin, amoeba lysates) [31]. These studies 
$\mathbf{a}$

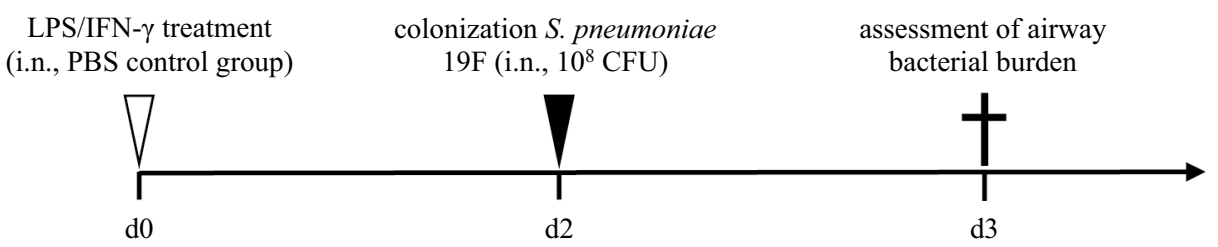

b

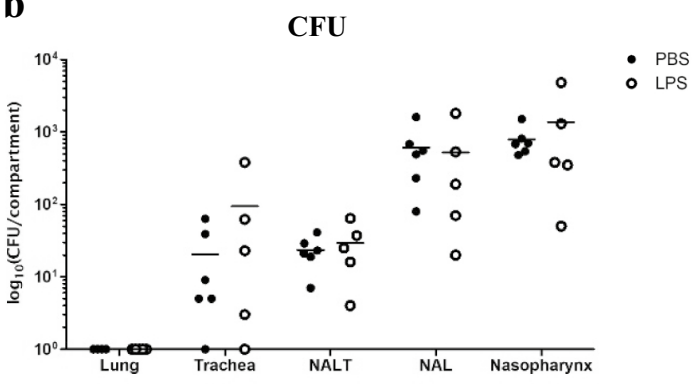

c

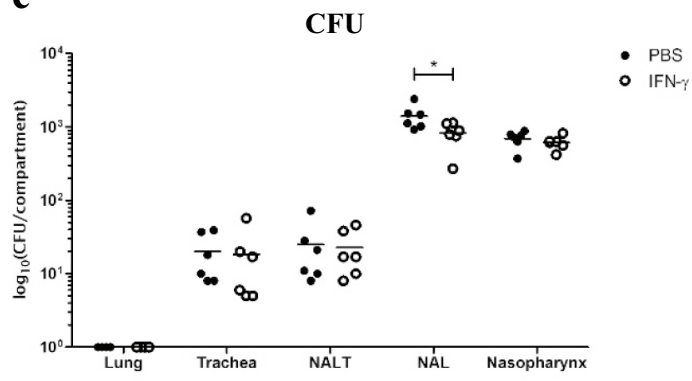

Fig. 5 Effect of LPS or IFN- $\gamma$ treatment on pneumococcal colonization in vivo. a Schematic representation of the experimental setup. b C57BL/6JRj mice were treated i.n. with $10 \mu \mathrm{g}$ of LPS $(n=5)$ and control mice received PBS only $(n=6)$. c C57BL/6JRj mice were treated i.n. with $1 \mu \mathrm{g}$ of IFN- $\gamma(n=6)$ and control mice received PBS

revealed that PIGR/Pigr expression is considerably influenced by exogenous and endogenous stimuli present in the airway microenvironment.

To our knowledge, we are first to report compartmentspecific and sex-independent differences in basal airway Pigr gene expression levels in vivo. We observed that BALB/c as well as $\mathrm{C} 57 \mathrm{BL} / 6 \mathrm{~J}$ mice showed highest Pigr expression in trachea, followed by NALT and lung. We speculated that this originated from inherent differences in microbial density. Due to their anatomical localization, trachea and nasal cavities are more frequently exposed to microbial stimuli compared to the lung [32]. It is possible that a higher abundance of microbial ligands in the murine URT provide more signals triggering Pigr gene expression compared to the LRT, as well. This in turn might prevent bacterial spread from URT to LRT contributing to the relatively low bacterial density in the lung. Contrary to our expectations, we could not detect any alterations regarding Pigr expression between germ-free and microbially colonized mice, which suggests that Pigr expression in the murine respiratory tract is in fact unaffected by microbial colonization. However, as we assessed whole-tissue Pigr gene expression, we cannot fully exclude the possibility that signal dilution effects $(e . g$., from leukocytes) affect compartment-specific Pigr expression in our analyses.

Commensal intestinal bacteria induce the production of $\operatorname{IgA}$ in mice [20]. Furthermore, it is known that lymphocyte numbers in nasal mucosa are dependent on housing conditions and exposure to microbial stimuli [33]. While those only $(n=6) .2$ days post-treatment all mice were i.n. infected with $10^{8} \mathrm{CFU}$ of $S$. pneumoniae $19 \mathrm{~F}$ and airway bacterial burden was assessed $18 \mathrm{~h}$ post-infection (individual data from one experiment are graphed; mean is indicated by horizontal line; * for $p \leq 0.05$ )

findings clearly highlight the impact of the microbiota on lymphocyte-associated mucosal immunity, the relationship between airway-associated secretory immunity and the level of microbial exposure is largely unknown. Our experiments revealed that Pigr expression was lower in NALT of mice with an undefined microbiome (highest microbial exposure) compared to mice maintained under SPF conditions. At the same time, airway and systemic IgA levels were increased in these mice. As fecal IgA levels depend on the composition of the intestinal microflora [34], it is conceivable that a similar effect might be present in murine airways. However, the fact that Pigr expression in NALT is reduced while IgA levels are increased indicates that there is likely no correlation between Pigr expression and IgA abundance at the wholetissue level. However, we did not analyze Pigr expression exclusively in stromal cells but in whole tissue. Since it was shown that the microbial environment shapes cell composition in the mucosa, it is possible that an accumulation of leukocytes in mice with a high microbial exposure reduces the overall Pigr signal.

Previous studies revealed that human and murine intestinal epithelial cells exhibit increased Pigr/PIGR gene expression after LPS stimulation in vitro [22, 35, 36]. In line with this, we detected significantly increased pulmonary Pigr expression after LPS treatment. However, we also found that LPS did not alter overall Pigr expression in trachea or NALT. This might arise from the fact that bacterial colonization-and therefore exposure to $e$.g., LPS-is more pronounced in the URT [32], resulting in a lower sensitivity of 
URT airway stromal cells to LPS. In contrast to this result, airway IgA levels were decreased in LPS-treated mice, while systemic IgA levels were unaffected. We hypothesized that decreased IgA levels resulted from decreased epithelial leakage and tested this by determining $\mathrm{Cldn} 18$ and $\mathrm{Cldn} 7$ expression. Claudins are major proteins that maintain epithelial barrier function and altered claudin expression results in altered AEC barrier function in the LRT ( Cldn18) and URT (Cldn7) [2-4]. However, Cldn18 and Cldn7 expression were not affected by LPS, which disconfirmed our hypothesis. Since IgA binds LPS [37], it is possible that the administered LPS was already bound to IgA in the mucosal lumen. This might reduce the amount of detectable $\operatorname{IgA}$, as the ELISA detects free IgA molecules with the highest functionality. However, the fact that Pigr expression in lung was increased while $\operatorname{IgA}$ in BAL was decreased and Pigr expression in NALT was unaltered while IgA in NAL was increased highlights that there is no consistent correlation between the two molecules in whole-tissue analysis of LPS-treated mice.

As LPS, IFN- $\gamma$ induces PIGR expression in human epithelial cells [23, 38, 39]; however, its effect on Pigr and secretory immunity in vivo have not been addressed before. Despite no effect on airway Pigr expression, IFN- $\gamma$ treatment increased pulmonary IgA levels. As Cldn expression was unaltered, we hypothesized that increased IgA concentrations after IFN- $\gamma$ treatment might arise from mucosal B cells. Yet, airway $I g J$ expression was not affected, which suggests that activated B cells are most likely not the cause of increased airway IgA levels following IFN- $\gamma$ stimulation. As mentioned before, we analyzed Pigr gene expression in tissues and not exclusively in stromal cells. It is known that intradermal IFN- $\gamma$ injection stimulates intradermal lymphocyte migration in rats [40]. Thus, it is conceivable that i.n. IFN- $\gamma$ treatment leads to the accumulation (and activation) of lymphocytes in the airways as well, which might reduce net Pigr expression. This might explain why there is no consistent correlation between Pigr expression and IgA levels in whole-tissue analysis of IFN- $\gamma$-treated mice.

As IgA is crucial for antimicrobial defense [15, 41-43], we investigated whether altered IgA levels upon LPS- and IFN- $\gamma$ treatment correlated with altered antimicrobial immunity. We have chosen $S$. pneumoniae for experimental colonization of mice as it is one of the most relevant respiratory pathogens [44] and $\operatorname{IgA}$ is vital for antagonizing pneumococcal colonization and infection in vivo and in vitro [12, 45-47]. Indeed, prophylactic IFN- $\gamma$ treatment significantly reduced nasal pneumococcal counts indicating improved antibacterial immunity. Since IFN- $\gamma$ treatment led to increased $\operatorname{IgA}$ levels only in BAL and not in NAL, the effect IFN- $\gamma$ has on colonization is most likely not mediated by its effect on IgA. Since IFN- $\gamma$ triggers antibacterial activity in pulmonary macrophages [48] and macrophages are present in the murine NALT [49], it is conceivable that
IFN- $\gamma$ induces antibacterial activity in these cells as well. IFN- $\gamma$ triggers the production of antibacterial molecules (e.g., $\beta$-defensins) [50]. Future studies will clarify whether IFN- $\gamma$-stimulated production of these antibacterial factors underlies the improved mucosal immunity in the URT.

In conclusion, our study demonstrates that secretory immunity in URT and LRT is differentially regulated by endogenous as well as exogenous stimuli. Further studies are needed to elucidate the underlying molecular frameworks as well as possible avenues for, e.g., prophylactic enhancement of airway mucosal immunity in infection-prone individuals.

Supplementary Information The online version contains supplementary material available at https://doi.org/10.1007/s00408-021-00498-8.

Authors' Contributions Conceptualization: AP, DB, and JDB; Methodology: AP and JF; Formal analysis and investigation: AP and JF; Writing — original draft preparation: AP; Writing — review and editing: DB and JDB; Funding acquisition: DB and JDB; Resources: KS, JS, and TS; Supervision: DB and JDB.

Funding Open Access funding enabled and organized by Projekt DEAL. This work was funded by the federal state Saxony-Anhalt and the European Structural and Investment Funds (ESF, 2014-2020), project number P4410032038.

Data Availability All data of this manuscript can be made available by the corresponding author.

Code Availability Not applicable.

\section{Declarations}

\section{Conflict of interest None.}

Ethical Approval The experiments were approved by the federal ethical bodies and were carried out in accordance with the guidelines of the Saxony-Anhalt State Administration Office and the guidelines of the Lower Saxony State Office for Consumer Protection and Food Safety.

Consent to Participate Not applicable.

Consent for Publication Not applicable.

Open Access This article is licensed under a Creative Commons Attribution 4.0 International License, which permits use, sharing, adaptation, distribution and reproduction in any medium or format, as long as you give appropriate credit to the original author(s) and the source, provide a link to the Creative Commons licence, and indicate if changes were made. The images or other third party material in this article are included in the article's Creative Commons licence, unless indicated otherwise in a credit line to the material. If material is not included in the article's Creative Commons licence and your intended use is not permitted by statutory regulation or exceeds the permitted use, you will need to obtain permission directly from the copyright holder. To view a copy of this licence, visit http://creativecommons.org/licenses/by/4.0/. 


\section{References}

1. Gumbiner B (1987) Structure, biochemistry, and assembly of epithelial tight junctions. Am J Physiol 253(6 Pt 1):C749-C758. https://doi.org/10.1152/ajpcell.1987.253.6.C749

2. Tsukita S, Furuse M (1999) Occludin and claudins in tightjunction strands: leading or supporting players? Trends Cell Biol 9(7):268-273. https://doi.org/10.1016/S0962-8924(99)01578-0

3. LaFemina MJ, Sutherland KM, Bentley T et al (2014) Claudin-18 deficiency results in alveolar barrier dysfunction and impaired alveologenesis in mice. Am J Respir Cell Mol Biol 51(4):550 558. https://doi.org/10.1165/rcmb.2013-0456OC

4. Clarke TB, Francella N, Huegel A et al (2011) Invasive bacterial pathogens exploit TLR-mediated downregulation of tight junction components to facilitate translocation across the epithelium. Cell Host Microbe 9(5):404-414. https://doi.org/10.1016/j.chom.2011. 04.012

5. Mills PR, Davies RJ, Devalia JL (1999) Airway epithelial cells, cytokines, and pollutants. Am J Respir Crit Care Med 160(5 Pt 2):S38-43. https://doi.org/10.1164/ajrccm.160.supplement_1.11

6. Bals R (2000) Epithelial antimicrobial peptides in host defense against infection. Respir Res 1(3):141-150. https://doi.org/10. $1186 / \mathrm{rr} 25$

7. Varsano S, Frolkis I, Rashkovsky L et al (1996) Protection of human nasal respiratory epithelium from complement-mediated lysis by cell-membrane regulators of complement activation. Am J Respir Cell Mol Biol 15(6):731-737. https://doi.org/10.1165/ ajrcmb.15.6.8969267

8. Kulkarni HS, Liszewski MK, Brody SL et al (2018) The complement system in the airway epithelium: an overlooked host defense mechanism and therapeutic target? J Allergy Clin Immunol 141(5):1582-1586.e1. https://doi.org/10.1016/j.jaci.2017.11. 046

9. Stegemann-Koniszewski S, Jeron A, Gereke M et al (2016) Alveolar Type II epithelial cells contribute to the anti-influenza a virus response in the lung by integrating pathogen- and microenvironment-derived signals. mBio. https://doi.org/10.1128/mBio. 00276-16

10. Phalipon A, Corthésy B (2003) Novel functions of the polymeric Ig receptor: well beyond transport of immunoglobulins. Trends Immunol 24(2):55-58. https://doi.org/10.1016/S1471-4906(02) 00031-5

11. Pilette C, Ouadrhiri Y, Godding V et al (2001) Lung mucosal immunity: immunoglobulin-A revisited. Eur Respir J 18(3):571588. https://doi.org/10.1183/09031936.01.00228801

12. Fukuyama Y, King JD, Kataoka K et al (2010) Secretory-IgA antibodies play an important role in the immunity to Streptococcus pneumoniae. J Immunol (Baltimore, Md.” 1950) 185(3):17551762. https://doi.org/10.4049/jimmunol.1000831

13. Tjärnlund A, Rodríguez A, Cardona P et al (2006) Polymeric IgR knockout mice are more susceptible to mycobacterial infections in the respiratory tract than wild-type mice. Int Immunol 18(5):807816. https://doi.org/10.1093/intimm/dx1017

14. Richmond BW, Brucker RM, Han W et al (2016) Airway bacteria drive a progressive COPD-like phenotype in mice with polymeric immunoglobulin receptor deficiency. Nat Commun. https://doi. org/10.1038/ncomms 11240

15. Polosukhin VV, Richmond BW, Du R et al (2017) Secretory IgA deficiency in individual small airways is associated with persistent inflammation and remodeling. Am J Respir Crit Care Med 195(8):1010-1021. https://doi.org/10.1164/rccm.201604-0759OC

16. Gohy ST, Detry BR, Lecocq M et al (2014) Polymeric immunoglobulin receptor down-regulation in chronic obstructive pulmonary disease. Persistence in the cultured epithelium and role of transforming growth factor- $\beta$. Am J Respir Crit Care Med. https:// doi.org/10.1164/rccm.201311-1971OC

17. Ladjemi MZ, Gras D, Dupasquier S et al (2018) Bronchial epithelial IgA secretion is impaired in asthma. Role of IL-4/IL-13. Am J Respir Crit Care Med. https://doi.org/10.1164/rccm. 201703-0561OC

18. Sandgren A, Albiger B, Orihuela CJ et al (2005) Virulence in mice of pneumococcal clonal types with known invasive disease potential in humans. J Infect Dis 192(5):791-800. https://doi.org/ $10.1086 / 432513$

19. Stegemann-Koniszewski S, Gereke M, Orrskog S et al (2013) TLR7 contributes to the rapid progression but not to the overall fatal outcome of secondary pneumococcal disease following influenza A virus infection. J Innate Immun 5(1):84-96. https:// doi.org/10.1159/000345112

20. Macpherson AJ, Uhr T (2004) Induction of protective IgA by intestinal dendritic cells carrying commensal bacteria. Science (New York, N.Y.) 303(5664):1662-1665. https://doi.org/10. 1126/science. 1091334

21. Hooper LV, Wong MH, Thelin A et al (2001) Molecular analysis of commensal host-microbial relationships in the intestine. Science (New York, N.Y.) 291(5505):881-884. https://doi.org/10. 1126/science.291.5505.881

22. Bruno MEC, Frantz AL, Rogier EW et al (2011) Regulation of the polymeric immunoglobulin receptor by the classical and alternative NF- $\mathrm{KB}$ pathways in intestinal epithelial cells. Mucosal Immunol 4(4):468-478. https://doi.org/10.1038/mi. 2011.8

23. Piskurich JF, France JA, Tamer CM et al (1993) Interferon- $\gamma$ induces polymeric immunoglobulin receptor mrna in human intestinal epithelial cells by a protein synthesis dependent mechanism. Mol Immunol 30(4):413-421. https://doi.org/10.1016/01615890(93)90071-I

24. Khattar NH, Lele SM, Kaetzel CS (2005) Down-regulation of the polymeric immunoglobulin receptor in non-small cell lung carcinoma: correlation with dysregulated expression of the transcription factors USF and AP2. J Biomed Sci 12(1):65-77. https:// doi.org/10.1007/s11373-004-8185-5

25. Loman S, Jansen HM, Out TA et al (1999) Interleukin-4 and interferon-gamma synergistically increase secretory component gene expression, but are additive in stimulating secretory immunoglobulin A release by Calu-3 airway epithelial cells. Immunology 96(4):537-543. https://doi.org/10.1046/j.1365-2567.1999. 00731.x

26. Polosukhin VV, Cates JM, Lawson WE et al (2011) Bronchial secretory immunoglobulin a deficiency correlates with airway inflammation and progression of chronic obstructive pulmonary disease. Am J Respir Crit Care Med 184(3):317-327. https://doi. org/10.1164/rccm.201010-1629OC

27. Pilette C, Godding V, Kiss R et al (2001) Reduced epithelial expression of secretory component in small airways correlates with airflow obstruction in chronic obstructive pulmonary disease. Am J Respir Crit Care Med 163(1):185-194. https://doi.org/10. 1164/ajrccm.163.1.9912137

28. Hupin C, Rombaux P, Bowen H et al (2013) Downregulation of polymeric immunoglobulin receptor and secretory IgA antibodies in eosinophilic upper airway diseases. Allergy 68(12):1589-1597. https://doi.org/10.1111/all.12274

29. Lewis CC, Aronow B, Hutton J et al (2009) Unique and overlapping gene expression patterns driven by IL-4 and IL-13 in the mouse lung. J Allergy Clin Immunol 123(4):795-804.e8. https:// doi.org/10.1016/j.jaci.2009.01.003

30. Jaffar Z, Ferrini ME, Herritt LA et al (2009) Cutting edge: lung mucosal Th17-mediated responses induce polymeric Ig receptor expression by the airway epithelium and elevate secretory $\operatorname{IgA}$ 
levels. J Immunol (Baltimore, Md.: 1950) 182(8):4507-4511. https://doi.org/10.4049/jimmunol.0900237

31. Carrasco-Yepez M, Campos-Rodriguez R, Lopez-Reyes I et al (2014) Intranasal coadministration of Cholera toxin with amoeba lysates modulates the secretion of IgA and IgG antibodies, production of cytokines and expression of pIgR in the nasal cavity of mice in the model of Naegleria fowleri meningoencephalitis. Exp Parasitol 145(Suppl):S84-92. https://doi.org/10.1016/j.exppa ra.2014.04.002

32. Man WH, de Steenhuijsen-Piters WAA, Bogaert D (2017) The microbiota of the respiratory tract: gatekeeper to respiratory health. Nat Rev Microbiol 15(5):259-270. https://doi.org/10.1038/ nrmicro.2017.14

33. Ichimiya I, Kawauchi H, Tanaka T et al (1991) Distribution of immunocompetent cells in normal nasal mucosa: comparisons among germ-free, specific pathogen-free, and conventional mice. Ann Otol Rhinol Laryngol 100(8):638-642. https://doi.org/10. 1177/000348949110000807

34. Ohashi Y, Hiraguchi M, Ushida K (2006) The composition of intestinal bacteria affects the level of luminal IgA. Biosci Biotechnol Biochem 70(12):3031-3035. https://doi.org/10.1271/bbb. 60164

35. Schneeman TA, Bruno MEC, Schjerven H et al (2005) Regulation of the polymeric Ig receptor by signaling through TLRs 3 and 4: linking innate and adaptive immune responses. J Immunol (Baltimore, Md.: 1950) 175(1):376-384. https://doi.org/10.4049/ jimmunol.175.1.376

36. Moon C, VanDussen KL, Miyoshi H et al (2014) Development of a primary mouse intestinal epithelial cell monolayer culture system to evaluate factors that modulate IgA transcytosis. Mucosal Immunol 7(4):818-828. https://doi.org/10.1038/mi.2013.98

37. Lüllau E, Heyse S, Vogel H et al (1996) Antigen binding properties of purified immunoglobulin A and reconstituted secretory immunoglobulin A antibodies. J Biol Chem 271(27):1630016309. https://doi.org/10.1074/jbc.271.27.16300

38. Nihei Y, Maruyama K, Endo Y et al (1996) Secretory component (polymeric immunoglobulin receptor) expression on human keratinocytes by stimulation with interferon- $\gamma$ and differences in response. J Dermatol Sci 11(3):214-222. https://doi.org/10.1016/ 0923-1811(95)00444-0

39. Loman S, Radl J, Jansen HM et al (1997) Vectorial transcytosis of dimeric IgA by the Calu-3 human lung epithelial cell line: upregulation by IFN-gamma. Am J Physiol 272(5 Pt 1):L951L958. https://doi.org/10.1152/ajplung.1997.272.5.L951

40. Issekutz TB, Stoltz JM, vd Meide P (1988) Lymphocyte recruitment in delayed-type hypersensitivity. The role of IFN-gamma. J Immunol (Baltimore, Md.: 1950) 140(9):2989-2993
41. Cunningham-Rundles C (2001) Physiology of IgA and IgA deficiency. J Clin Immunol 21(5):303-309. https://doi.org/10.1023/A: 1012241117984

42. Edwards E, Razvi S, Cunningham-Rundles C (2004) IgA deficiency: clinical correlates and responses to pneumococcal vaccine. Clin Immunol (Orlando, Fla.) 111(1):93-97. https://doi.org/10. 1016/j.clim.2003.12.005

43. Janzi M, Kull I, Sjöberg R et al (2009) Selective IgA deficiency in early life: association to infections and allergic diseases during childhood. Clin Immunol (Orlando, Fla.) 133(1):78-85. https:// doi.org/10.1016/j.clim.2009.05.014

44. Catterall JR (1999) Streptococcus pneumoniae. Thorax 54(10):929-937. https://doi.org/10.1136/thx.54.10.929

45. Sun K, Johansen F, Eckmann L et al (2004) An important role for polymeric Ig receptor-mediated transport of IgA in protection against Streptococcus pneumoniae nasopharyngeal carriage. J Immunol (Baltimore, Md.: 1950) 173(7):4576-4581. https://doi. org/10.4049/jimmunol.173.7.4576

46. Sestini P, Nencioni L, Villa L et al (1988) IgA-driven antibacterial activity against Streptococcus pneumoniae by mouse lung lymphocytes. Am Rev Respir Dis 137(1):138-143. https://doi. org/10.1164/ajrccm/137.1.138

47. Fasching CE, Grossman T, Corthésy B et al (2007) Impact of the molecular form of immunoglobulin A on functional activity in defense against Streptococcus pneumoniae. Infect Immun 75(4):1801-1810. https://doi.org/10.1128/IAI.01758-06

48. Jensen WA, Rose RM, Burke RH Jr et al (1988) Cytokine activation of antibacterial activity in human pulmonary macrophages: comparison of recombinant interferon- $\gamma$ and granulocyte-macrophage colony-stimulating factor. Cell Immunol 117(2):369-377. https://doi.org/10.1016/0008-8749(88)90126-8

49. Heritage PL, Underdown BJ, Arsenault AL et al (1997) Comparison of murine nasal-associated lymphoid tissue and Peyer's patches. Am J Respir Crit Care Med 156(4 Pt 1):1256-1262. https://doi.org/10.1164/ajrccm.156.4.97-03017

50. Albanesi C, Fairchild HR, Madonna S et al (2007) IL-4 and IL-13 negatively regulate TNF-alpha- and IFN-gamma-induced betadefensin expression through STAT-6, suppressor of cytokine signaling (SOCS)-1, and SOCS-3. J Immunol (Baltimore, Md.: 1950) 179(2):984-992. https://doi.org/10.4049/jimmunol.179.2.984

Publisher's Note Springer Nature remains neutral with regard to jurisdictional claims in published maps and institutional affiliations. 